\title{
Energy Scavenging for Sensor Applications using Structural Strains
}

\author{
Joshua P. Ayers ${ }^{\mathrm{a}}$, David W. Greve ${ }^{\mathrm{b}}$, Irving J. Oppenheim ${ }^{\mathrm{c}^{*}}$ \\ a Department of Mechanical Engineering \\ ${ }^{\mathrm{b}}$ Department of Electrical and Computer Engineering \\ ${ }^{c}$ Department of Civil and Environmental Engineering \\ Carnegie Mellon University
}

\begin{abstract}
Resident sensors are envisioned for civil infrastructure applications, but providing long-term power for such devices remains a design challenge. An ideal solution would be to scavenge energy from structural strains and make the scavenging component a part of the sensor package. In principle, piezoelectric materials are suited to that role, and studies by others have demonstrated the feasibility of energy scavenging from flexible PZT devices operated at large strains and high strain rates. We have conducted experiments to collect electrical energy from PZT ceramics. We summarize the governing piezoelectric equations and outline the most convenient forms to use for the energy scavenging problem, illustrated by tracing one complete loading cycle. We review the material properties for the three PZT ceramics used in our experiments. We show experimental results recording voltage and charge in the cases of open-circuit, resistive loads, and capacitive loads, showing good agreement with analytical predictions. However, the greatest challenge is the approach to energy storage. In theory, capacitors can store energy but at varying voltage and with non-negligible leakage, whereas a battery can store energy at constant voltage with little leakage. We conducted experiments on both approaches, and we discuss our findings of the feasibility and efficiency of battery recharging at the scale of our devices, which have nominal dimensions of 10x10x1 mm.
\end{abstract}

Keywords: Piezoelectric, PZT, scavenging, strain energy.

\section{INTRODUCTION}

Piezoelectric devices are potentially useful for scavenging electrical power from time-varying strains in structures. This power can be used to operate resident sensors that would periodically perform measurements, store data, and report by RF communications when polled. The candidate application discussed here is to scavenge energy from structural strains using PZT (lead-zirconium-titanate, a piezoceramic) specimens with mm-scale dimensions in thickness and $\mathrm{cm}$-scale dimensions in length and width.

The device proposed must satisfy these requirements:

- The device must be powered by changes in strain regularly encountered in a structure.

- The device must be sufficiently small to form part of the packaging of an intended sensor.

- The device must provide sufficient power for the intended periodic use of the sensor.

Our preliminary analysis suggests that these requirements can be satisfied. The simple case studied here is a thin disc polarized in the through-thickness direction, for which a mechanical change in thickness creates a voltage across those same poling electrodes. Because the experimental study of piezoelectric performance may be new to many researchers in the field of structures, in this paper we first present the relevant background theory and then we report results from our laboratory investigations.

\footnotetext{
* Contact author: ijo@cmu.edu; 412-268-2950; Carnegie Mellon University, Department of Civil and Environmental Engineering, Pittsburgh, PA 15213.
} 


\section{PREVIOUS WORK}

The direct piezoelectric effect is the development of surface electrical charge in response to mechanical stress, and was first demonstrated as a property of certain asymmetric crystals by Pierre and Jacques Curie in 1880. The inverse piezoelectric effect, the development of mechanical strain (dimensional change) in response to an applied electrical field, was predicted by Lippmann in 1891 and then quickly confirmed by the Curies. The crystallographic principles were described by Voigt in 1910. An early application was the first ultrasonic submarine sensors developed by Langevin in 1917, and another was the resonance of piezoelectric crystals for frequency control. Piezoelectricity remains the basis for many excitation and sensing capabilities including most ultrasonic and ultrasound applications. The reader is referred to the relevant chapter ${ }^{1}$ in the text by Mason $^{2}$ and to a paper by Ballato ${ }^{3}$ for an introduction to piezoelectricity.

The use of the direct piezoelectric effect for power scavenging is the subject of significant recent attention. One project at MIT $^{4}$ involved the development of footwear heel materials containing a piezoelectric layer for the purpose of powering electronic devices carried by personnel. A recent laboratory study at Sandia ${ }^{5}$ was a successful integrated demonstration of the use of power scavenging, structural sensing, and communications within a prospective application of building surety. The study used a commercial PZT bending transducer, a microcontroller, and an RFID device to demonstrate energy conversion under high flexural strain, in which the microcontroller was powered and the RFID signal was encoded. Ottman et. al. ${ }^{6}$ recently described a piezoelectric generator under sinusoidally varying strain, showed analytical and experimental results comparable to the ones presented here, and studied the use of a voltage controller to increase charging efficiency.

\section{BACKGROUND}

\subsection{Overview of piezoelectricity and development of the governing equations}

In an elastic material, equations relate the stress and strain tensors denoted $\{\mathrm{T}\}$ and $\{\mathrm{S}\}$, respectively. In a dielectric material, equations relate the electric field and electric displacement tensors, denoted $\{E\}$ and $\{D\}$, respectively. In a piezoelectric material the quantities are coupled to one another. In principle, one pair of coupled equations should suffice for a typical application. However, the piezoelectric equations have conventionally been developed to map out all possible pairings of dependent and independent variables, and as a result four pairs of coupled equations are generally encountered. The four pairs are, of course, equivalent and can be derived from one another. However, the alternate forms of the material constants needed for each equation pair, and the alternate forms by which material constants are reported by manufacturers, add to the burden of using these equations. We present all four pair of equations, and material constants, for the case of a specific PZT material. The matrices described below are simplified using the particular case of the PZT crystal symmetries.

\subsection{Reference axes and stress/strain definitions}

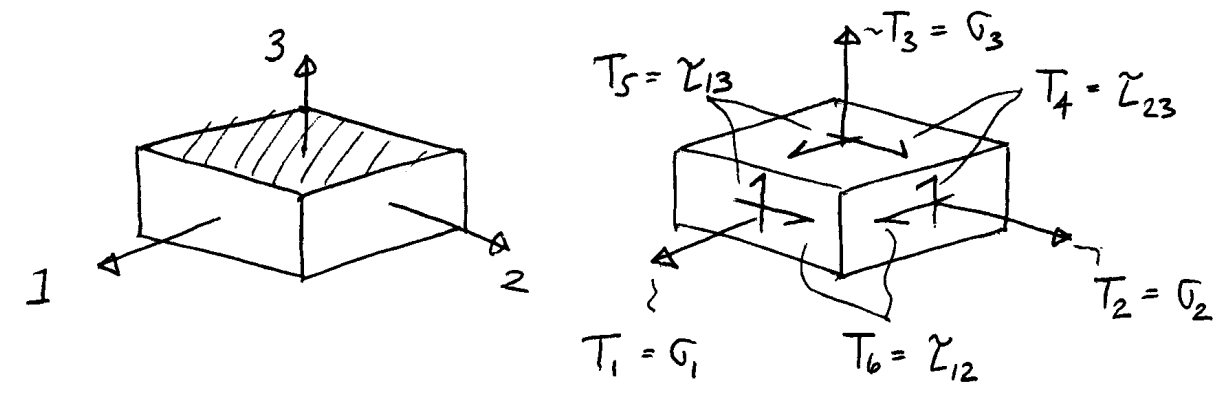

Figure 1. Reference axes for stress components

The piezoelectric properties of a PZT are formed by electroding two parallel surfaces and poling the material between them. The direction of poling is defined as the 3-axis, and two orthogonal directions are defined as the 1-axis and 2axis. For convenience we will work with rectangular cuboids and we will set the 1-axis and 2-axis to be parallel to the 
edges of those specimens, as shown in Figure 1. Stresses are shown on the visible surfaces with the familiar symbols of $\sigma$ for normal stress and $\tau$ for shear stress. In the piezoelectric equations the six stress components are defined as $\mathrm{T}_{1}$ through $\mathrm{T}_{6}$, as shown; components $\mathrm{T}_{1}$ through $\mathrm{T}_{3}$ correspond to normal stresses, while $\mathrm{T}_{4}$ through $\mathrm{T}_{6}$ correspond to shear stresses. The strain components $S_{1}$ through $S_{6}$ are analogous. Stress $\{T\}$ and strain $\{S\}$ are both vectors of length 6 .

In the absence of piezoelectric behavior coupling the mechanical and electrical variables, the elastic relation between strain and stress, or between stress and strain, is simply:

$$
\{\mathrm{T}\}=[\mathrm{c}]\{\mathrm{S}\} \quad \text { or } \quad\{\mathrm{S}\}=[\mathrm{s}]\{\mathrm{T}\}
$$

where $[c]$ is a $6 \times 6$ stiffness matrix and $[\mathrm{s}]$ is a $6 \times 6$ compliance matrix. Matrices $[\mathrm{c}]$ and $[\mathrm{s}]$ are inverse to one another.

In a piezoelectric material, the stiffness (and compliance) properties are different under constant electric field than they are under constant electric displacement. Accordingly, the [c] and [s] matrices must be defined with respect to those two conditions, indicated by superscripts E and D, as follows:

$$
\left[c^{\mathrm{E}}\right]=\text { stiffness matrix at constant electric field }
$$

with $\left[\mathrm{c}^{\mathrm{D}}\right],\left[\mathrm{s}^{\mathrm{E}}\right]$, and $\left[\mathrm{s}^{\mathrm{D}}\right]$ accordingly.

The PZT materials are members of a specific crystal class ${ }^{3}$ (labeled $6 \mathrm{~mm}$, or $6_{\mathrm{Cv}}$, or dihexagonal pyramidal, or Class 26) possessing important symmetries. The material properties are symmetrical about the 3 -axis, which is why we may conveniently assign the 1-axis and 2-axis parallel to the sides of our specimen. Whereas a general anisotropic material has 21 coefficients in its [c] or [s] matrix, materials with the PZT crystal structure have only four independent coefficients. Moreover, many stress/strain pairs are not coupled to one another, reflected by matrix coefficients equal to zero. The form for the $[\mathrm{c}]$ matrix for this class is as follows:

$$
\begin{array}{cccccc}
c_{11} & c_{12} & c_{12} & 0 & 0 & 0 \\
c_{12} & c_{11} & c_{12} & 0 & 0 & 0 \\
c_{12} & c_{12} & c_{33} & 0 & 0 & 0 \\
0 & 0 & 0 & c_{44} & 0 & 0 \\
0 & 0 & 0 & 0 & c_{44} & 0 \\
0 & 0 & 0 & 0 & 0 & \frac{c_{11}-c_{12}}{2}
\end{array}
$$

Coefficient $\mathrm{c}_{66}$ is sometimes listed separately, but is not independent of $\mathrm{c}_{11}$ and $\mathrm{c}_{12}$, as shown. Matrix [s] is analogous except that $\mathrm{s}_{66}$ is equal to $2\left(\mathrm{~s}_{11}-\mathrm{s}_{12}\right)$.

\subsection{Dielectric properties}

The electric properties of interest, electric field $\mathrm{E}$ and electric displacement $\mathrm{D}$, may have components along each of the three principal axes. Therefore, electric field $\{E\}$ and electric displacement $\{D\}$ are vectors of length 3 . In the absence of piezoelectric behavior that couples the mechanical and electrical conditions, the relation between electric displacement and electric field would be written $\{E\}=[\varepsilon]\{D\}$, where $[\varepsilon]$ is the permittivity, most commonly expressed as a dielectric constant relative to $\varepsilon_{0}$, the permittivity of free space. In this formulation, $[\mathcal{E}$ ] would be a $3 \times 3$ matrix. The inverse relation between electric field and electric displacement would be written $\{\mathrm{D}\}=[\beta]\{\mathrm{E}\}$, where impermeability $[\beta]$ is also a $3 \times 3$ matrix, and where $[\varepsilon]$ and $[\beta]$ are the inverse of one another. For PZT the $[\varepsilon$ ] matrix is diagonal with only two independent coefficients: $\varepsilon_{11}$ (which equals $\varepsilon_{22}$ ) and $\varepsilon_{33}$. The [ $\beta$ ] matrix is similarly diagonal, each term being the reciprocal of the corresponding term in the $[\varepsilon]$ matrix. 
In a piezoelectric material, the permittivity (and impermeability) properties depend upon whether the electric field and electric displacement occur under constant stress or under constant strain. Accordingly, the $[\varepsilon]$ and $[\beta]$ matrices must be defined under those two conditions, indicated by superscripts $\mathrm{T}$ and $\mathrm{S}$. Therefore, $\left[\varepsilon^{\mathrm{T}}\right]$ is the permittivity matrix at constant stress, with $\left[\varepsilon^{\mathrm{S}}\right],\left[\beta^{\mathrm{T}}\right]$, and $\left[\beta^{\mathrm{S}}\right]$ defined accordingly.

\subsection{Piezoelectric matrices}

The piezoelectric properties relate the six mechanical terms to the three electrical terms, or vice versa, and therefore will take the form of a $3 \times 6$ (or $6 \times 3$ ) matrix. The piezoelectric properties are variously recorded as the $[\mathrm{d}],[\mathrm{e}],[\mathrm{g}]$, or $[\mathrm{h}]$ matrices. Referring to the [d] matrix, a PZT has only three independent coefficients. Moreover, along many of the direction pairings there is no coupling, reflected by matrix coefficients equal to zero. The form of matrix [d] for this crystal class is as follows:

$$
\begin{array}{cccccc}
0 & 0 & 0 & 0 & d_{15} & 0 \\
0 & 0 & 0 & d_{15} & 0 & 0 \\
d_{31} & d_{31} & d_{33} & 0 & 0 & 0
\end{array}
$$

Matrices [e], $[\mathrm{g}]$, and $[\mathrm{h}]$ are analogous. The matrices are not independent and can be derived ${ }^{3}$ from one another. Quantities in [d], [e], [g], and [h] have units of $\mathrm{c} / \mathrm{N}, \mathrm{c} / \mathrm{m}^{2}, \mathrm{Vm} / \mathrm{N}$, and $\mathrm{V} / \mathrm{m}$, respectively. The quantity $\mathrm{d}_{33}$ is sometimes termed the piezoelectric modulus, $\mathrm{g}_{33}$ the piezoelectric pressure constant, and $\mathrm{h}_{33}$ the piezoelectric deformation constant. Note that the units of $g$ are equivalent to V/m (electric field) per Pascal.

\subsection{Governing equations, coupling factors}

The governing equations are grouped below into four pairs. A matrix transpose is denoted with a prime.

$$
\begin{aligned}
& \{T\}=\left[c^{E}\right]\{S\}-\left[e^{\prime}\right]\{E\} \\
& \{D\}=[e]\{S\}+\left[\varepsilon^{S}\right]\{E\} \\
& \{T\}=\left[c^{D}\right]\{S\}-\left[h^{\prime}\right]\{D\} \\
& \{E\}=-[h]\{S\}+\left[\beta^{S}\right]\{D\} \\
& \{S\}=\left[s^{E}\right]\{T\}+\left[d^{\prime}\right]\{E\} \\
& \{D\}=[d]\{T\}+\left[\varepsilon^{T}\right]\{E\} \\
& \{S\}=\left[s^{D}\right]\{T\}+\left[g^{\prime}\right]\{D\} \\
& \{E\}=-[g]\{T\}+\left[\beta^{T}\right]\{D\}
\end{aligned}
$$

Piezoelectric material properties are most commonly characterized with the use of coupling factors, expressing the ratio of the mutual energy to the geometric mean of the elastic energy and the dielectric energy. For a one-dimensional stress state $\mathrm{T}_{3}$ (with all other stresses equal to zero) the coupling factor is designated $\mathrm{k}_{33}$ and is shown in Mason ${ }^{1}$ to equal: 


$$
k_{33}=\frac{d_{33}}{\sqrt{\varepsilon^{T}{ }_{33} \cdot s^{E}{ }_{33}}}
$$

For strain $S_{3}$, with all other strains equal to zero, the coupling factor is designated $\mathrm{k}_{\mathrm{t}}$ and is equal to:

$$
k_{t}=\frac{h_{33}}{\sqrt{c^{D}{ }_{33} \cdot \beta^{S}{ }_{33}}}
$$

In practice, piezoelectric material properties are generally expressed with a small number of individual constants and a well-chosen set of coupling factors.

\section{ANALYSIS OF A FOUR-STEP LOAD AND DISCHARGE CYCLE}

It is instructive to discuss a four-step cycle of load, discharge, unload, and discharge. In our example calculation we use published material properties for PZT-5 taken from Mason ${ }^{1,2}$, and we apply and remove a uni-axial compressive stress by applying a force of $1 \mathrm{kN}$ to a specimen measuring $10 \mathrm{~mm} \times 25 \mathrm{~mm} \times 1 \mathrm{~mm}$. The four-step cycle proceeds as follows:

1. Stress $T_{3}$ (equal to $-4 \mathrm{MPa}$ ) is applied and we use Eq's. 4 to find strain $\{S\}$ and electric field $\{E\}$. Strain $S_{3}$ is calculated as $\mathrm{s}_{33} \mathrm{~T}_{3}$ and equals $-38 \mu$ e. Electric field $\mathrm{E}_{3}$ is calculated as $-\mathrm{g}_{33} \mathrm{~T}_{3}$ and equals $99 \mathrm{kV} / \mathrm{m}$, and is multiplied by the thickness, $1 \mathrm{~mm}$, to obtain a voltage of $99 \mathrm{~V}$.

2. The device is discharged; we allow current to flow between the electrodes until $\{E\}$ is equal to zero and then we use Eqs. 3 to find strain $\{S\}$ and electric displacement $\{D\}$. A new strain $S_{3}$ is calculated as $\mathrm{s}_{33}^{\mathrm{E}} \mathrm{T}_{3}$ and equals $-75 \mu$ e; the magnitude of compressive strain has increased as the electric charge was drawn. Electric displacement $D_{3}$ is calculated as $d_{33} T_{3}$ and equals $-0.0015 \mathrm{coul} / \mathrm{m}^{2}$.

3. $\mathrm{T}$ is removed and we use Eqs. 4. A new strain $\mathrm{S}_{3}$ is calculated as $\mathrm{g}_{33} \mathrm{D}_{3}$ and equals $-37 \mu$ e. Electric field $\mathrm{E}_{3}$ is calculated as $\beta^{\mathrm{T}}{ }_{33} \mathrm{D}_{3}$ and equals $-99 \mathrm{kV} / \mathrm{m}$. The magnitude of compressive strain has decreased as the stress was removed, and the electric field is opposite in sign as compared to step 1.

4. The device is discharged, setting $\{\mathrm{E}\}$ to zero. All quantities return to zero, illustrating that the compressive strain is not recovered until the remaining electric charge is drawn.

Mechanical work is done by the applied load in steps 1 and 2, and is only partly recovered from strain energy when unloaded in step 3. The "loss" in mechanical work is accounted for by the drawing of electrical energy from the system. Similarly, an outside force must work to "lift" the weight in step 4, or else the system cannot return to its zero position.

\section{EXPERIMENTAL RESULTS}

\subsection{Preliminary study of electrical properties}

In our experiments we used PZT specimens of $0.66 \mathrm{~mm}$ thickness, $6.45 \mathrm{~mm}$ width, and $6.45,12.9$, or $19.4 \mathrm{~mm}$ length, fabricated from three different PZT materials (designated A, B, C) with properties given in Table 1. The piezoelectric pressure constant, $\mathrm{g}_{33}$, is seen to decrease as the dielectric constant increases.

\begin{tabular}{|l|l|l|l|l|l|}
\hline Type & Dielectric constant $\left(\varepsilon_{33}^{\mathrm{T}}\right.$, relative $)$ & $\mathrm{d}_{33}, \mathrm{c} / \mathrm{N}$ & $\mathrm{g}_{33}, \mathrm{Vm} / \mathrm{N}$ & $\mathrm{k}_{33}$ & $\mathrm{k}_{\mathrm{t}}$ \\
\hline $\mathrm{A}$ & 425 & $150 \times 10^{-12}$ & $39.9 \times 10^{-3}$ & 0.66 & 0.50 \\
\hline B & 1300 & $295 \times 10^{-12}$ & $25.0 \times 10^{-3}$ & ---- & --- \\
\hline C & 3800 & $650 \times 10^{-12}$ & $19.0 \times 10^{-3}$ & 0.75 & 0.55 \\
\hline
\end{tabular}

Table 1. Properties of three piezoelectric materials studied experimentally

An unstressed specimen functions electrically as a parallel plate capacitor with capacitance $\mathrm{C}$ calculated as

$$
C=\frac{A \varepsilon}{h}
$$


where $\mathrm{A}$ is the plate area, $\mathrm{h}$ is the PZT thickness or the plate spacing, and $\varepsilon$ is obtained by multiplying $\varepsilon_{0}$ (the permittivity of free space, $8.85 \times 10^{-12} \mathrm{c}^{2} / \mathrm{Nm}^{2}$ ) by the relative dielectric constant $\varepsilon^{\mathrm{T}}{ }_{33}$. Specimens with three different areas were prepared from each of the three PZT products and the resulting sample capacitances were measured at 1 MHz. The measured capacitance was proportional to area, and the dielectric constants extracted from a linear fit were 452, 1326, and 3443 for A, B, and C respectively.

We then conducted a series of experiments measuring voltage as a function of time under various conditions of electrical load. Data was obtained using a DAQ plug-in card controlled by NI-DAQ/Labview software. A National Instruments SCXI-1122 16 channel isolated transducer multiplexer module was used for signal conditioning. The multiplexer presents a resistive load to the PZT capacitor which tends to discharge the capacitor. With no other load, the capacitor voltage is given by

$$
V(t)=V_{0} e^{-t / R C}
$$

where $\mathrm{C}$ is the PZT capacitance and $\mathrm{R}$ is the input resistance of the multiplexer. The multiplexer gain was set to a specified input resistance of $1 \mathrm{G} \Omega$, yielding a predicted discharge time constant of $0.23 \mathrm{sec}$ for the smallest specimen of material A. The discharge time was further shortened by leakage effects, and therefore in subsequent experiments we used larger specimens of materials B and C because they exhibited lower leakage effects and longer time constants.

\subsection{Derivation of the Equivalent Circuit Model}

We can easily show that the PZT under load acts as a current source in parallel with a capacitor. Considering a uni-axial stress state in the 3-direction and omitting the subscripts, one of the governing equations for piezoelectric material is

$$
D=d T+\varepsilon^{T} E
$$

where $\mathrm{D}$ is electric displacement, $\mathrm{T}$ is stress, and $\mathrm{E}$ is electric field.

Taking the derivative with respect to time yields

$$
\frac{\partial D}{\partial t}=d \frac{\partial T}{\partial t}+\varepsilon^{T} \frac{\partial E}{\partial t}
$$

In this expression, the time derivative of the electric displacement is related to the external current through

$$
\frac{\partial D}{\partial t}=\frac{i}{A_{e}}
$$

where $\mathrm{i}$ is the current and $\mathrm{A}_{\mathrm{e}}$ is the electrical area, the area of the PZT where a charge is developed. The time derivative of the stress is given by

$$
\frac{\partial T}{\partial t}=\frac{1}{A_{m}} \frac{\partial F}{\partial t}
$$

where $\mathrm{F}$ is the force applied and $\mathrm{A}_{\mathrm{m}}$ is the mechanical area. Finally, assuming a constant electric field in the bulk of the PZT, we have

$$
\varepsilon^{T} \frac{\partial E}{\partial t}=\frac{\varepsilon^{T}}{h} \frac{\partial V}{\partial t}
$$

where $\mathrm{V}$ is the voltage across the PZT and $\mathrm{h}$ is the thickness of the PZT.

Substituting Eqs. 10, 11, and 12 into 3b and using Eq. 7 for a parallel plate capacitor, we obtain

$$
i=d \frac{A_{e}}{A_{m}} \frac{\partial F}{\partial t}+C \frac{\partial V}{\partial t}
$$

The first term is proportional to the rate at which load is applied, and the second term is the current through a capacitor. The interpretation of this result is that a piezoelectric material acts like a current source in parallel with a capacitor. The capacitance has the value of the PZT capacitance and the current source output is proportional to the rate at which the external load is applied. 


\subsection{Verification of the model}

Several simple experiments were used to verify this result, using the circuit shown below. The PZT is represented by the parallel combination of a current source and a capacitor.

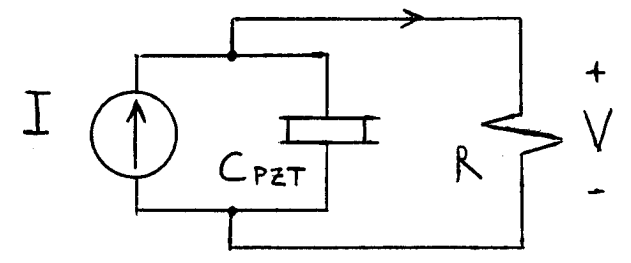

Figure 2. Circuit representing PZT and resistive load

The transfer function from the current I to the voltage across the resistor is

$$
\frac{V}{I}=\frac{R}{s C R+1}
$$

where s is the Laplace transform complex frequency. Resistors of $10 \mathrm{M} \Omega$ and $20 \mathrm{M} \Omega$ were connected across the PZT specimen, which was loaded by an Instron 4400R testing machine. The voltage was sampled at $100 \mathrm{~Hz}$ and the load was sampled at $10 \mathrm{~Hz}$. The load data was interpolated using a cubic spline and numerically differentiated, and the current was then calculated using the discrete time equation, derived from Eq. 13,

$$
i=d \frac{A_{e}}{A_{m}} \frac{\Delta F}{\Delta t}
$$

The predicted voltage was determined using the Matlab LSIM command and the transfer function (Eq. 14). Figure 3 shows the predicted and measured voltages from an experiment in which the load was increased linearly to $1 \mathrm{kN}$ over a $2.5 \mathrm{sec}$ period and then held constant, with electrical discharge into a resistive load. Figure 4 shows the predicted and measured voltages from an experiment in which the load was cycled between 0 and $1 \mathrm{kN}$ following a triangular (sawtooth) history at a $4 \mathrm{sec}$ period. The predicted and measured voltages show good agreement, validating our model.
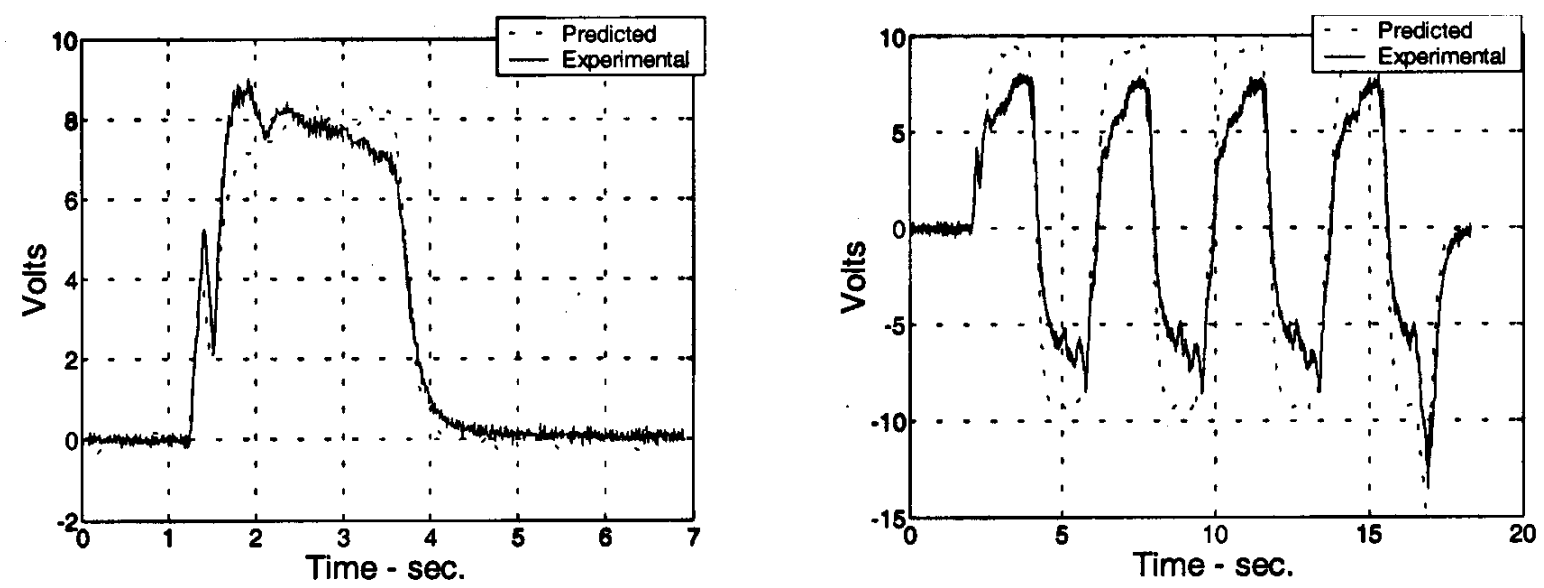

Figure 3. Type B PZT discharging to a $20 \mathrm{M} \Omega$ resistor. Figure 4. Type C PZT discharging to a $10 \mathrm{M} \Omega$ resistor, cyclic loading. 


\subsection{Charging a capacitor}

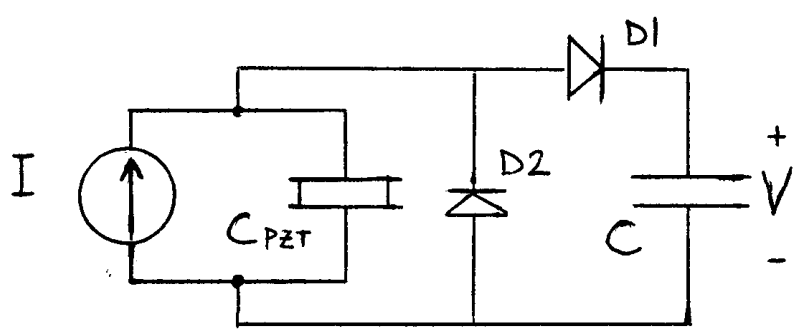

Figure 5. Circuit employed in charging experiments

We next consider charging an external capacitor using the circuit of Figure 5. The PZT is connected to the charging circuit and subjected to force $\mathrm{F}(\mathrm{t})$, with $\mathrm{F}(0)$ equal to zero and $\mathrm{F}\left(\mathrm{t}_{2}\right)$ denoting the maximum force applied. The voltage on the external capacitor at the start of the loading cycle is $\mathrm{V}_{0}$ and (neglecting the diode turn-on voltage) diode D1 turns on when the PZT voltage reaches this value. For $0 \leq t \leq t_{1}$ diode D1 is off; and for $t_{1} \leq t \leq t_{2}$ diode D1 is on and the external capacitor is being charged.

The current output may be expressed as

$$
i(t)=d_{33} \frac{A_{e}}{A_{m}} \frac{\partial F}{\partial t}
$$

For $0 \leq t \leq t_{1}, V(t)=\frac{Q(t)}{C_{p z t}}$, where the capacitor charge, $Q(t)$ may be calculated as

$$
Q(t)=\int_{0}^{t} I(\tau) d \tau=\int_{0}^{t} d_{33} \frac{A_{e}}{A_{m}} \frac{\partial F}{\partial t} d \tau=\frac{A_{e}}{A_{m}} d_{33} \cdot F(t)
$$

At $t_{1}$, the diode just turns on and we have

$$
V\left(t_{1}\right)=V_{o}=\frac{Q\left(t_{1}\right)}{C_{p z t}}=\frac{d_{33} A_{e} / A_{m} F\left(t_{1}\right)}{C_{p z t}}
$$

rearranging and solving for $F\left(t_{1}\right)$

$$
F\left(t_{1}\right)=\frac{V_{o} C_{p z t}}{d_{33} A_{e} / A_{m}}
$$

For $t_{1} \leq t \leq t_{2}$, the PZT voltage is

$$
V(t)=V_{0}+\frac{Q\left(t-t_{1}\right)}{C_{p z t}+C}
$$

where

$$
Q\left(t-t_{1}\right)=\int_{t_{1}}^{t} I(\tau) d \tau=d_{33} \frac{A_{e}}{A_{m}}\left(F(t)-F\left(t_{1}\right)\right)
$$


Substituting Eqs. 19 and 21 into Eq. 20 and rearranging yields the voltage at the end of the charging period

$$
V\left(t_{2}\right)=V_{o}\left(1-\frac{C_{p z t}}{C_{p z t}+C}\right)+\frac{d_{33} A_{e} / A_{m} F\left(t_{2}\right)}{C_{p z t}+C}
$$

which is applied incrementally to obtain the voltage after each loading cycle.

Experiments were performed using two PZT specimens (material B, $6.45 \mathrm{~mm}$ x $19.4 \mathrm{~mm}$, the size having been chosen for ease of handling), in parallel electrically and in series structurally such that $A_{e} / A_{m}$ is 2 , loaded cyclically to $1 \mathrm{kN}$ following a triangular (sawtooth) pattern at $0.66 \mathrm{~Hz}$. Figures 6 and 7 show the measured and predicted voltages. The increase in voltage $\mathrm{V}$ is essentially linear with the number of cycles.

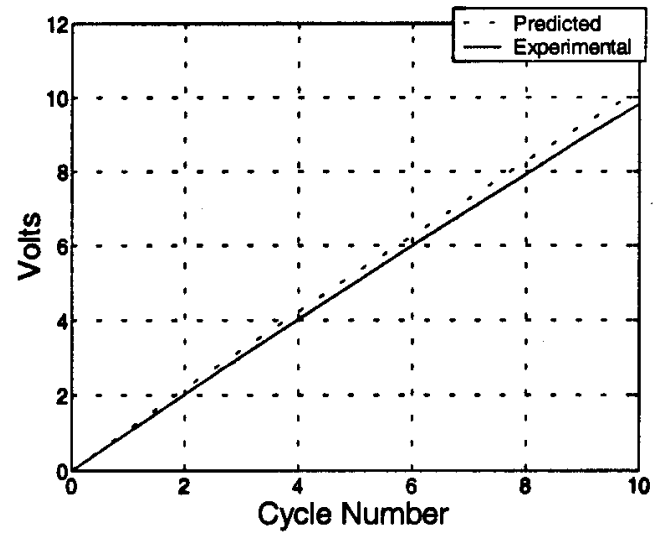

Figure 6. Charging a $1 \mathrm{uF}$ capacitor

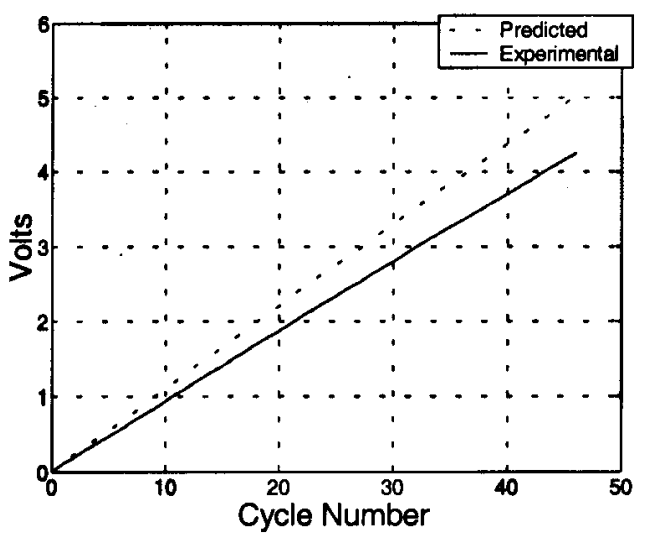

Figure 7. Charging a $10 \mathrm{uF}$ capacitor

The energy stored in the capacitor at any time is calculated as $\mathrm{E}=1 / 2 \mathrm{CV}^{2}$, with $\mathrm{V}$ obtained from Eq. 22 . Under uniform cyclical forcing, we find that maximum energy storage in a single cycle is obtained when the capacitor is at a voltage

$$
V_{o p t}=\frac{\left(1-\frac{C_{p z t}}{C_{p z t}+C}\right) \cdot\left(\frac{d_{33} A_{e} / A_{m} F\left(t_{2}\right)}{C_{p z t}+C}\right)}{2\left[1-\left(1-\frac{C_{p z t}}{C_{p z t}+C}\right)^{2}\right]}
$$

Although this result is interesting, it is of limited practical significance for power scavenging from structural strains because the loads, such as axle loads from trucks on highway bridge girders, will vary.

\subsection{Charging a battery}

Finally, we consider a cycle in which an electrochemical battery is charged at constant voltage, with the equivalent circuit depicted in Figure 8. The derivation is similar to that in section 5.4 for charging a capacitor. Again, the load F is applied to the PZT from time 0 to time $t_{2}$. Diode D1 turns on at time $t_{1}$ and until the time that diode turns on there is no difference between this case and that of the capacitor, so Eqs. 16 through 20 are still valid. For $t_{1} \leq t \leq t_{2}$, $V(t)=V_{o}$ since the battery voltage remains constant during the charging cycle. Combining this with Eq. 16 yields an expression for power supplied to the battery 


$$
P(t)=V(t) \cdot i(t)=V_{o} d_{33} \frac{A_{e}}{A_{m}} \frac{\partial F}{\partial t}
$$

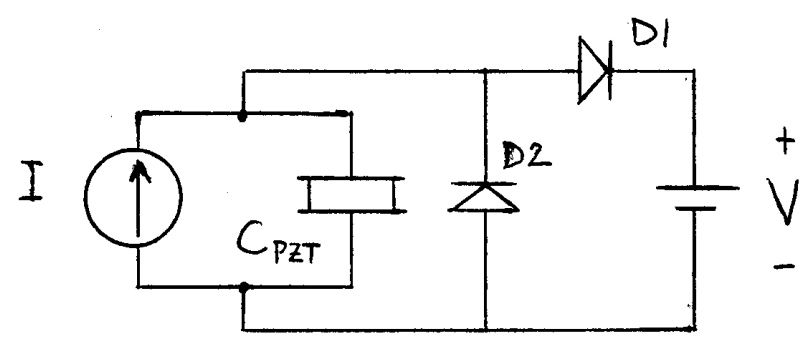

Figure 8. Circuit for battery charging

The total energy stored in one cycle is

$$
W=\int_{t_{1}}^{t_{2}} P(\tau) d \tau=\int_{t_{1}}^{t_{2}} V_{o} d_{33} \frac{A_{e}}{A_{m}} \frac{\partial F}{\partial t} d \tau=V_{o} d_{33} \frac{A_{e}}{A_{m}}\left(F\left(t_{2}\right)-F\left(t_{1}\right)\right)
$$

Substituting Eq. 19 for $F\left(t_{1}\right)$ yields the energy W, per cycle, as

$$
W=V_{o} d_{33} \frac{A_{e}}{A_{m}} F\left(t_{2}\right)-V_{o}^{2} C_{p z t}
$$

This result suggests that the PZT capacitance should be as small as possible. This is reasonable, because the larger the PZT capacitance, the more charge is wasted bringing the PZT voltage up to the battery voltage during each cycle.

Experiments were performed using four PZT specimens (material B, $6.45 \mathrm{~mm}$ x $19.4 \mathrm{~mm}$ ), in parallel electrically and in series structurally such that $A_{e} / A_{m}$ is 4 , loaded cyclically to $1 \mathrm{kN}$ following a triangular (sawtooth) pattern at $0.66 \mathrm{~Hz}$. The energy charged a Panasonic ML616 rechargeable battery, which has a nominal capacity of roughly 30 J. Figure 9 shows the measured battery open-circuit voltage increasing over the course of 92,000 load cycles, establishing that the battery received charge; charging was also confirmed by applying a $270 \mathrm{k} \Omega$ resistive load to the battery, measuring a $2.17 \mathrm{~V}$ drop before charging and $2.25 \mathrm{~V}$ after charging. Approximately $0.86 \mathrm{~J}$ of energy was transferred to the battery.

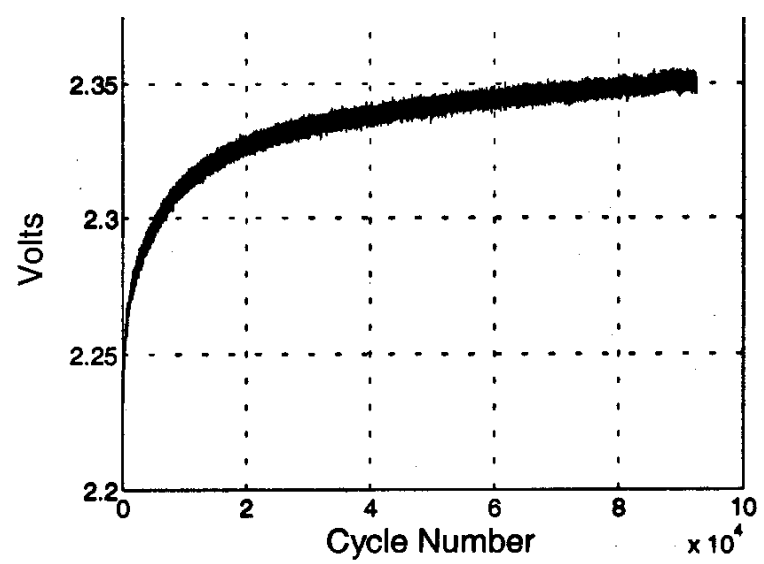

Figure 9. Battery voltage increase during charging, 92000 cycles 
Using Eq. 26, the battery voltage that gives optimum energy storage can be calculated. Taking the partial derivative of Eq. 26 with respect to $\mathrm{V}_{0}$ and setting it to zero gives

$$
\begin{aligned}
\frac{\partial W}{\partial V_{o}} & =d_{33} \frac{A_{e}}{A_{m}} F\left(t_{2}\right)-2 V_{o} C_{p z t}=0 \\
V_{o p t} & =\frac{A_{e}}{A_{m}} \frac{d_{33} F\left(t_{2}\right)}{2 C_{p z t}}
\end{aligned}
$$

An upper bound to the energy that can be extracted in any cycle is the amount of energy stored in the PZT capacitor were it not connected to an external circuit. Using Eq. 17, the charge as a function of applied load may be calculated as

$$
Q_{\max }=\frac{A_{e}}{A_{m}} d_{33} \cdot F
$$

From this result, the electrical energy stored in the PZT is

$$
W_{\max }=\frac{1}{2} C_{p z t} V_{\max }^{2}=\frac{1}{2} \frac{Q_{\max }{ }^{2}}{C_{p z t}}
$$

Substituting Eq. 29 into Eq. 30 yields

$$
W_{\max }=\frac{1}{2 C_{p z t}}\left(\frac{A_{e}}{A_{m}} d_{33} \cdot F\right)^{2}
$$

This result suggests, in theory, an optimum force F at which a PZT might be operated.

We finally substitute the optimum voltage $\mathrm{V}_{\text {opt }}$, Eq. 28, into the expression for the energy stored in a battery, Eq. 26:

$$
W_{o p t}=\frac{1}{2 C_{p z t}}\left(\frac{A_{e}}{A_{m}} d_{33} \cdot F\right)^{2}-\frac{1}{4 C_{p z t}}\left(\frac{A_{e}}{A_{m}} d_{33} \cdot F\right)^{2}
$$

Upon manipulation we obtain:

$$
W_{o p t}=\frac{1}{4 C_{p z t}}\left(\frac{A_{e}}{A_{m}} d_{33} \cdot F\right)^{2}
$$

Comparing Eq. 33 with Eq. 31, we observe

$$
W_{o p t}=\frac{1}{2} W_{\max }
$$




\section{DISCUSSION AND CONCLUSIONS}

A background discussion of piezoelectric charge generation and energy storage was presented. Equivalent circuit models were examined for discharge, for charging a capacitor, and for charging a battery. Experiments were performed to verify and demonstrate each case. The laboratory experiments were performed as imposed-force loadings, inducing a strain of roughly $70 \mu \mathrm{e}$. Within the stress range and specimen dimensions studied, on the order of $10^{5}$ cycles would be needed to transfer $1 \mathrm{~J}$ of electrical energy.

Our experiments were performed as imposed-force loadings, and not as imposed-strain loadings which would apply for specimens affixed to structural members. Packaging of specimens and their coupling to structural members would constitute an additional engineering problem, which has not been addressed. However, coupling specimens to regions of highest cyclical strain could increase the charge developed in the PZT.

As presented in Eqs. 28-34, if a PZT is operated optimally with the battery voltage satisfying Eq, 28, the energy delivered to the battery in each cycle is one-half the energy stored in the PZT. Proper matching of the battery voltage to the PZT will therefore improve charging efficiency. However, that optimal voltage will typically be much higher than the voltages at which sensors operate. For example, for the loads and PZT properties used in our experiments, the optimum battery voltage would be around 60 volts; in prospective applications for power scavenging from structural strains, the optimum battery voltage could be higher. One possible approach for future work would be to charge many batteries in series, so as to approach an optimum voltage level, and to switch the batteries into parallel for powering sensors at lower voltages.

\section{ACKNOWLEDGEMENTS}

This work was supported by the National Science Foundation through the Research Experience for Undergraduates program as part of grant CMS-9980759, A Micro-NMR Device for In-situ Detection of Chlorides in Concrete. The Institute for Complex Engineering Systems at Carnegie Mellon University provided valuable assistance, both in support of this research and in support of the REU mission. Krautkramer (Agfa NDT) supplied and fabricated the PZT specimens and provided technical guidance to the research team. The authors gratefully acknowledge all the instances of support for this effort.

\section{REFERENCES}

1. Berlincourt, D. A., Curran, D. R., and Jaffe, H., Piezoelectric and Piezomagnetic Materials and Their Function in Transducers, Chapter 3, 169-270, of reference 2, 1964.

2. Mason, W. P. (editor), Physical Acoustics; Principles and Methods, Volume $1-$ Part A; $4^{\text {th }}$ edition, Academic Press, New York, 1964..

3. Ballato, A., "Piezoelectricity: Old Effect, New Thrusts," IEEE Trans. On Ultrasonics, Ferroelectrics, and Frequency Control, Vol. 42, 916-926, 1995.

4. Schenck, N. S., and Paradiso, J. A., "Energy Scavenging with Shoe-Mounted Piezoelectrics," IEEE Micro, 30-42, May-June 2001.

5. Pfeifer, K. B., Leming, S. K., and Rumpf, A. N., Embedded Self-Powered Microsensors for Monitoring the Surety of Critical Buildings and Infrastructures, SAND2001-3619, Sandia National Laboratories, November 2001.

6. Ottman, G. K., Hofmann, H. F., Bhatt, A. C., and Lesieutre, G. A., "Adapative Piezoelectric Energy Harvesting Circuit for Wireless Remote Power Supply,” IEEE Trans. On Power Electronics, Vol. 17, 669-676, 2002. 